\title{
Atmospheres, magnetism, mass loss of red supergiant stars
}

\author{
E. Josselin ${ }^{1,2}$, J. Lambert ${ }^{2}$, M. Aurière ${ }^{3}$, P. Petit ${ }^{3}$ and N. Ryde ${ }^{2}$ \\ ${ }^{1}$ LUPM, Université Montpellier II , CNRS, France \\ email: eric.josselin@univ-montp2.fr \\ ${ }^{2}$ Department of Astronomy and Theoretical Physics, Lund Observatory, Sweden \\ ${ }^{3}$ Université de Toulouse, UPS-OMP, Institut de Recherche en Astrophysique et Planétologie, \\ Toulouse, France
}

\begin{abstract}
Red supergiant stars (RSGs) are not only a key evolutionary stage of massive stars participating in the chemical evolution of galaxies, they also represent a fantastic and challenging laboratory of (magneto-)hydrodynamics. We present recent results and on-going research on mass loss, atmospheres, and polarimetric studies of RSGs that reveal a magnetic field of unknown origin. We discuss the potential interplay between these different processes.
\end{abstract}

Keywords. Polarization, stars: atmospheres, stars: magnetic fields, stars: mass loss, supergiants

\section{Introduction}

Red supergiant stars (hereafter RSGs) are evolved massive stars in the core helium burning phase. They are characterized by high luminosities (of the order of $10^{5} \mathrm{~L}_{\odot}$ ) and rather low temperatures (3500-4000 K), thus being the biggest stars, reaching radii up to $\sim 1500 \mathrm{R}_{\odot}$ (Levesque et al. 2005).

Mass loss is a key-phenomenon in the evolution of RSGs, and of massive stars in general. In particular, it governs the blue-to-red ratio, the properties of subsequent WolfRayet stars, the location in the HR diagram of type II supernova progenitors, etc. (Mauron \& Josselin 2011). The process at the origin of these winds remains however unknown. It should be a hybrid process, since the velocity of ejection $\left(\sim 10-40 \mathrm{~km} \mathrm{~s}^{-1}\right)$ is supersonic but much slower than the escape velocity at the level of the photosphere. However, this process must be different from that invoked for thermally-pulsing asymptotic giant branch stars, which combines levitation of gas due to pulsations and radiation pressure on the subsequently formed dust. Indeed, RSGs are not regular pulsators, but rather low-amplitude irregular variables. Furthermore, observations have shown that dust condensation operates too far from the photosphere, typically $r_{\text {cond }} \gtrsim 20 R_{\star}$ (Danchi et al. 1994). Among the alternative explanations, Alfvén waves are quite often proposed as part of the solution.

\section{Magnetic fields in RSGs: linear polarization diagnostics}

Simultaneously with the work of Grunhut et al. (2010), who showed the common occurrence of magnetic fields at the (sub-)gauss level in F to $\mathrm{K}$ supergiants, Aurière et al. (2010) performed the first detection in an M-type supergiant, namely $\alpha$ Ori, with a longitudinal field of approximately 1 Gauss. It should be kept in mind that, in particular because of the very slow rotation rate of these stars, the total intensity and the geometry of these magnetic fields remain unknown. 
Can this field be generated by a standard dynamo process (esp. $\alpha \Omega$ )? This hypothesis is commonly tested through the computation of the Rossby number, Ro, i.e. the ratio of inertial to Coriolis forces (computed here as the ratio between the rotation period and the convective turnover timescale), which should be $\ll 1$ for a global dynamo process to be efficient (see Aurière et al. 2015). A rough estimation of Ro can be done according to

$$
R o \approx \frac{\tau_{\text {rot }}}{\tau_{\text {conv }}} \approx \tau_{\text {rot }} \times \frac{v_{\text {conv }}}{\alpha H_{p}}
$$

assuming that convection is well described by the 1D mixing length theory (MLT) approximation. In the case of $\alpha$ Ori, the rotation period is $\tau_{\text {rot }} \simeq 17$ years, the convective velocity is $v_{\text {conv }} \approx 20 \mathrm{~km} \mathrm{~s}^{-1}$ and the pressure scale height is $H_{p} \approx 0.1 R_{\star}$ (Schwarzschild 1975). This leads to $R o \sim 90$. It thus seems that RSGs are not able to sustain large-scale dynamos that can generate a global magnetic field. However, the large-scale convective motions may generate a small-scale dynamo action, and thus transitory fields.

Furthermore, $\alpha$ Ori exhibits a strong linear polarization signal in spectral lines, up to a few percents in the blue. This linear polarization, $p_{L}$, decreases with wavelength, and this decrease is compatible with $p_{L} \propto \lambda^{-4}$. We may thus observe depolarization of the continuum radiation, which is polarized by Rayleigh scattering, within spectral lines, because of collisions in the line formation process, or interferences between partially overlappping magnetic substates. The polarization of the continuum is linked with asymmetries generated by giant convective cells. Based on the $\mathrm{CO}^{5} \mathrm{BOLD}$ radiative hydrodynamics simulations (Freytag et al. 2012) and assuming pure Rayleigh scattering (and that the last scattering process dominates), we predict $p_{L} \approx 2 \%$ in the blue.

This linear polarization may thus constitute a potential diagnostic of photospheric asymmetries, and a new tool to measure magnetic fields, as long as we can infer the contribution of the Hanle effect on it.

\section{References}

Aurière, M., Donati, J.-F., Konstantinova-Antova, R., Perrin, G., Petit, P., \& Roudier, T. 2010, $A \mathscr{E} A$ 516, L2

Aurière, M., Konstantinova-Antova, R., Charbonnel, C., Wade, G. A., Tsvetkova, S., Petit, P., Dintrans, B., Drake, N. A., Decressin, T., Lagarde, N., and 8 coauthors 2015, A\& A 574, A90

Danchi, W. C., Bester, M., Greenhill, L. J., Degiacomi, C. G., Townes, C.H.1994, AJ 107, 1469

Freytag, B., Steffen, M., Ludwig, H.-G., Wedemeyer-Bhm, S., Schaffenberger, W., \& Steiner, O. 2012, J. Comp. Ph. 231, 919

Grunhut, J. H., Wade, G. A., Hanes, D. A., \& Alecian, E. 2010, MNRAS 408, 2290

Josselin, E. \& Plez, B. 2007, A\&\& 469, 671

Levesque, E. M., Massey, P., Olsen, K. A. G., Plez, B., Josselin, E., Maeder, A., \& Meynet, G. 2005, ApJ 628, 973

Mauron, N. \& Josselin, E. 2011, A\&A 526, 156

Schwarzschild, M. 1975, ApJ 195, 137 